\title{
Position of Experts Regarding Follow-Up of Patients with Neuronal Ceroid Lipofuscinosis-2 Disease in Latin America
}

Journal of Inborn Errors

of Metabolism \& Screening

2020, Volume 8: e20200012

DOI: https://doi.org/10.1590/2326-4594-

JIEMS-2020-0012

\author{
Norberto Guelbert ${ }^{1}$, Nora Atanacio ${ }^{2}$, Inés Denzler ${ }^{3}$, Emília Katiane \\ Embiruçu ${ }^{4}$, Nury Mancilla ${ }^{5}$, Ricardo Naranjo ${ }^{6} \oplus$, André Pessoa ${ }^{7}$, \\ Norma Spécola ${ }^{8}$, Lina Tavera ${ }^{9} \odot$, Mónica Troncoso ${ }^{10}$, Diane Vergara ${ }^{10}$
}

\begin{abstract}
Given the lack of standardized guidance for follow-up of patients with neuronal ceroid lipofucsinosis-2 disease in Latin-American countries and the heterogeneity of the region, an expert panel was created with the participation of 11 pediatric neurologists from Colombia, Argentina, Brazil and Chile. The aim of the expert panel was to describe a framework for standardized follow-up in patients with neuronal ceroid lipofucsinosis-2 disease, on or off therapy, that could benefit patients and treating physicians alike. Experts made recommendations in the following areas: seizures, abnormal movements and ataxia, sleep disorders and pain, cognitive function, visual function, hearing and speech, cardiac function, quality of life, and motor function. Recommendations include the most appropriate tools for use in the Latin-American context and health care systems, and provide feasible follow-up guidance, applicable in public and private healthcare facilities. They take into consideration the availability of clinical assessment resources, tools (scales, questionnaires, paraclinical tests) and access to these tools in Latin-American countries, as well as other regional and local needs defined by the participating experts.
\end{abstract}

\section{Keywords:}

CLN2 disease, neuronal ceroid lipofuscinosis type 2, follow-up, lipofuscin storage diseases, neuronal ceroid lipofuscinosis.

\section{Introduction}

Neuronal ceroid lipofucsinosis-2 (CLN2) disease (OMIM \# 204500), known as late infantile form, is a pediatric disease caused by intracellular fluorescent ceroid and lipofuscin-like deposits containing lipid peroxides and a subunit $\mathrm{c}$ of mitochondrial ATP synthase in different body tissues. It is characterized by rapidly progressing epilepsy due to the destruction of the central nervous system as a result of neuronal death related to lipofuscin accumulation in neuronal and glial cell lysosomes.

The spectrum of CLN2 disease comprises several clinical presentations: a) late infantile lipofuscinosis, b) juvenile form of CLN2 disease, and c) adult form of CLN2 disease. The "classic" phenotype of the disease is late infantile lipofuscinosis, and the "atypical" phenotype or late presentation refers to late onset and prolonged course of the disease $(1,2)$. The disease follows a devastatingly rapid course, with symptoms and loss of function advancing with age. The following is the timeline of symptom onset and loss of function in the classic form of CLN2 disease (Figure 1).

The initial clinical compromise in patients with CLN2 disease is characterized by language delay, epileptic seizures and ataxia ( 2 to 4 years of age), followed by cognitive and motor impairment
${ }^{1}$ Hospital de Niños de Córdoba, Departamento de Neurología Pediátrica, Córdoba, Argentina.

${ }^{2}$ Hospital de Niños Pedro de Elizalde, Departamento de Neurología Pediátrica, Buenos aires, Argentina.

${ }^{3}$ Hospital Italiano de Buenos Aires, Departamento de Neurología Pediátrica, Buenos Aires, Argentina.

${ }^{4}$ Hospital Santa Izabel, Departamento de Neurologia Pediátrica, Salvador, BA, Brazil.

${ }^{5}$ Hospital Universitario Clínica San Rafael, Departamento de Neurología Pediátrica, Bogotá, Colombia.

${ }^{6}$ Fundación Hospital de la Misericordia, Departamento de Neurología Pediátrica, Bogotá, Colombia.

${ }^{7}$ Universidade Estadual do Ceará - UECE, Hospital Infantil Albert Sabin, Departamento de Neurologia-Neurogenética, Fortaleza, CE, Brazil.

${ }^{8}$ Hospital de Niños de La Plata, Departamento de Neurología Pediátrica, La Plata, Buenos Aires, Argentina.

${ }^{9}$ Neuro Conexión, Departamento de Neurología Pediátrica, Armenia, Colombia. ${ }^{10}$ Universidad de Chile, Hospital San Borja Arriarán, Departamento de Neurología Pediátrica, Santiago, Chile.

Received August 20, 2020, and in revised form September 28, 2020. Accepted for publication October 1, 2020.

\section{Corresponding Author:}

Norberto Guelbert, Hospital de Niños de Córdoba, Departamento de Neurología Pediátrica, Córdoba, Argentina.

Email: nguelbert@gmail.com 


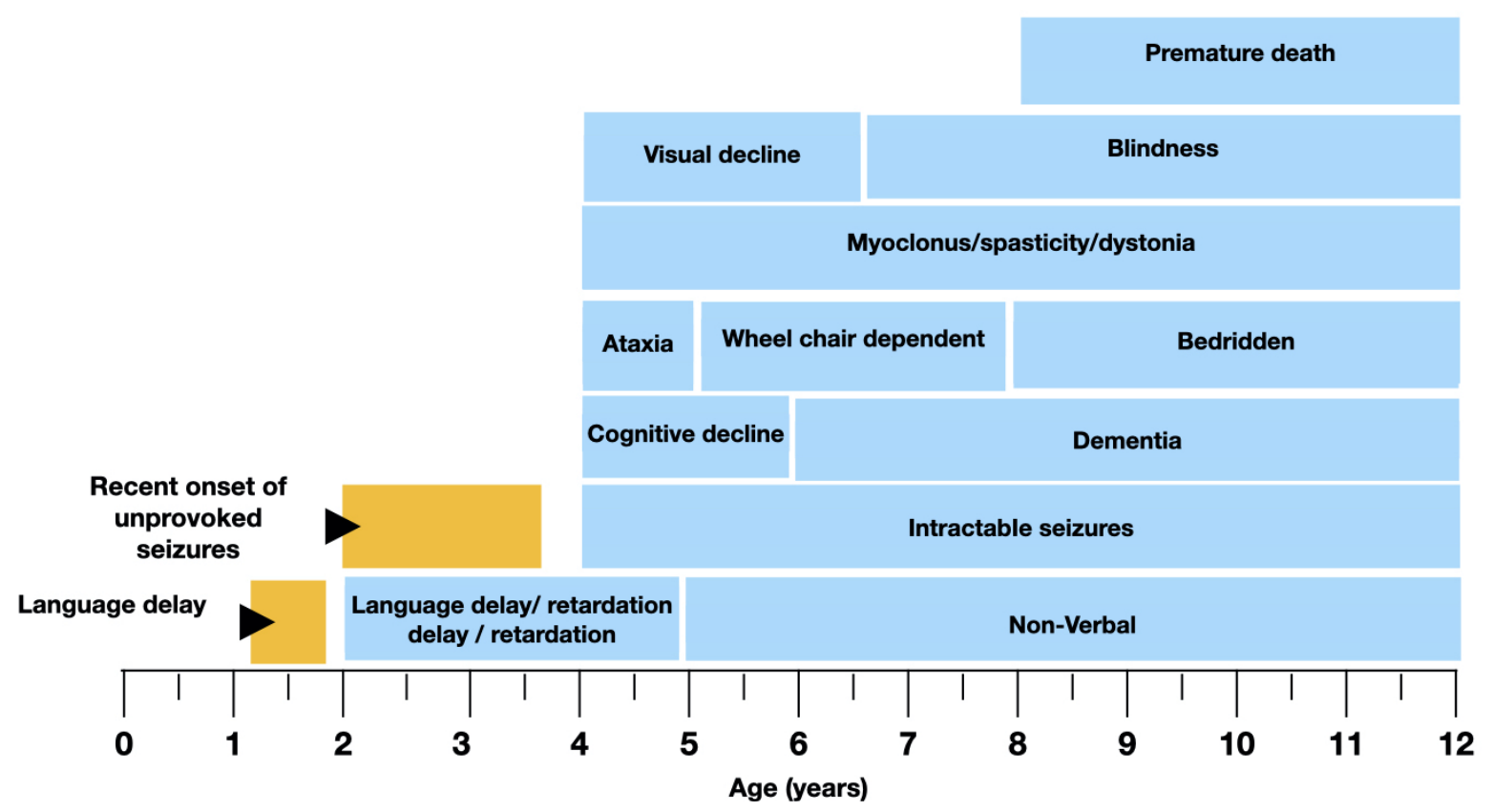

Figure 1. Symptom onset timeline. Taken from https://www.cln2connection.com/es-co/overview/natural-history/. (1-7).

with complete loss of motor function by 6 years of age, and death in mid-childhood.

CLN2 disease is a rare disease whose diagnosis, treatment and follow-up pose significant challenges for healthcare systems and providers, and create substantial barriers that patients and families need to overcome.

There is paucity of references on standardized recommendations for the follow-up of patients with CLN2 disease in the literature. (3)

This work analyzes various aspects that need to be considered when it comes to defining the most appropriate follow-up recommendations for patients with CLN2 disease in the Latin American context: results and recommendations derived from the available evidence, degree of sensitivity of the tests and their relevance in terms of applicability and reproducibility in different countries, availability of resources and clinical assessment tools (scales, questionnaires, paraclinical tests), access to these tools in public and private healthcare settings, and other needs and peculiarities of the region, as defined by the participating experts. The aim of this work was to describe a framework for standardized follow-up in patients with CLN2 disease, on or off therapy, based on expert recommendations.

\section{Methods}

\section{Identification of the Participants - Expert Panel}

A group of experts met on November 21, 2019. The group consisted of 11 pediatric neurologists: 3 from Colombia who attended the meeting in person, and the remaining 8 who participated online, 4 from Argentina, 2 from Brazil and 2 from Chile. The experts were selected based on their knowledge of the subject matter and their experience managing patients with CLN2 disease.

\section{Review of the Literature}

Before the meeting, the panel members conducted an independent review of the literature on the clinical manifestations of CLN2 disease and the follow-up tools used in the 9 areas in which the main clinical manifestations of the disease occur.

\section{Preparation of the Recommendations}

Each expert prepared recommendations in one area, by completing the questionnaire shown below. In those cases, in which there was no published evidence on the topic, recommendations were made based on experience.

- Clinical manifestations of CLN2 disease in the area for which you provide recommendations.

- Tools (scores, laboratory tests, images, etc.) recommended for use in patient follow-up, specifying grade of recommendation (mandatory, desirable, optional, not recommended), frequency, practitioner who must perform the test, practitioner in charge of follow-up, and validation of the tools used.

\section{Meetings to Define the Content of the Position Statement}

The expert panel met twice. The first meeting took place on site (August 2019) and the second took place online (November 2019). The recommendations were submitted and discussed in a plenary meeting until consensus was reached. The group 
then agreed on the final content of the Position Statement Paper regarding follow-up of patients with CLN2 disease in the Latin American region.

A facilitator gathered the information submitted by the members of the expert panel into a document that reflected the group's position. This document was put the consideration of all the panelists, who approved the final version for publication.

\section{Results}

Annex 1 shows the summary table of recommendations.

\section{Follow-up of seizures and loss of brain volume}

\section{Clinical manifestations}

In classic CLN2 disease cases, the age of onset of seizures is between 2 and 4 years. Seizures become refractory to treatment soon and patients end up receiving multiple medications (valproate, benzodiazepines). Seizures are polymorphic: myoclonic, generalized tonic-clonic, focal, absences and atonic. $(3,4)$ These patients may present altered electroencephalographic tracings such as an early positive photoparoxysmal response to low-frequency light stimulus (1-3 Hz). Electroencephalographic (EEG) changes appear as neurological decline progresses, with slowing of the baseline pattern and altered tracings. $(4,5)$ Magnetic resonance imaging (MRI) shows early onset progressive cerebellar atrophy, atrophy of cerebellar hemispheres, hyperintense periventricular white matter, progressive cerebral atrophy, and thalamic hypointensity. With progression, disseminated grey matter atrophy occurs due to neuronal loss. $(6,7)$

Secondary endpoint findings in the clinical study included slowing of brain volume loss on MRI, consistent with the expected pharmacological effect of intracerebroventricular enzyme replacement therapy. (8)

\section{Tools for patient follow-up}

\section{Clinical assessment}

- Frequency: At every visit

- Follow-up by: neurologist/pediatric neurologist

- Recommendation grade: Mandatory

- Observations: The course of the seizures (type of ictus, frequency), anticonvulsants used (name and dose) and the results of enzyme replacement therapy (ERT) assessed with clinical scores must all be documented in the clinical record.

\section{Electroencephalogram (EEG)}

- Frequency (experience-based): At baseline (presence of seizures), with follow-up every 6 months, according to the course of the disease. (7)

- Test performed by: EEG technician.

- Recommendation grade: Mandatory.
- Observations: Test to be conducted with the photostimulation equipment (starting at $1-3 \mathrm{~Hz}$, followed by $5-20 \mathrm{~Hz}$ and ending with high frequencies $>20 \mathrm{~Hz}$ ), (9) during waking hours with eyes open, at a distance of $30 \mathrm{~cm}$ from the photostimulation equipment. If the age of the patient does not allow it, the test must be performed during sleep.

\section{Magnetic Resonance Imaging (MRI)}

- Frequency: At baseline, with annual follow-up or according to the course of the disease. No annual followup in patients with a score of 0 on the Neuronal Ceroid Lipofuscinosis Type 2 (CLN2) Clinical Rating Scale (CLN2 scale), in a very advanced stage. (9)

- Test performed by: Technician

- Recommendation grade: Mandatory.

- Observations: MRI with epilepsy protocol, T1, T2, Flair sequences and enhanced water diffusion in the ADC technique. Spectroscopy to be done if possible. The use of gadolinium is not recommended. (6) MRI helps with decision-making regarding management and need for treatment, and allows to stratify patients in accordance with imaging progression.

\section{Follow-up of abnormal movements and ataxia}

\section{Clinical manifestations}

Children with CLN2 disease exhibit different types of movement disorders, the most important being ataxia, which is common in classic and also in the late presentation. Myoclonus (epileptic and non-epileptic) is characteristic, while dystonia and spasticity are commonly seen. Chorea, athetosis and tremors may also be present. Although rarely seen in the late infantile phenotype, parkinsonism, ataxia and prominent chorea have been reported in atypical phenotypes. (10-15)

\section{Recommended tools for use in patient follow-up}

\section{Clinical assessment}

- Frequency: At every visit

- Information to be gathered by: Neurologist/pediatric neurologist

- Recommendation grade: Mandatory

- Observations: Clinical follow-up is required when myoclonus, dystonia and other abnormal movements are present

\section{Scale for the Assessment of Rating Scale (SARA)}

- Frequency: At every visit (10 - 20 minutes)

- Scale to be administered by: Neurologist/pediatric neurologist or trained clinician/pediatrician

- Recommendation grade: Desirable

- Validation: Scale validated in Spain and Brazil

- Observations: The Hamburg, Weill Cornell and the Clinical Scoring System for Late Infantile Neuronal Ceroid Lipofuscinoses (LINCL) scales may be used 
to assess various impairments in cerebellar ataxia, but SARA is useful for a more sensitive and specific assessment because it is easily reproducible between examiners and provides an overview of axial and appendicular cerebellar coordination. The scale assesses a range of impairments in cerebellar ataxia. $(15,16)$

\section{Other scales}

- Although not mandatory, dystonia assessment could be made using specific scales such as the Burke-FahnMarsden (BFMDRS), used to assess dystonia severity in 9 regions of the body. (17) If the examiner lacks training/ experience using scales, a thorough clinical assessment must be performed (body segments where it occurs, at rest or in motion, or determination of whether it is triggered by a specific situation). (18)

\section{Follow-up of sleeping disorders and pain}

\section{Sleep: Clinical manifestations}

Sleep disorders in patients with epilepsy may be caused by the epileptic disorder itself or by the anticonvulsants. They appear early on, are difficult to manage and are a significant source of concern for the family. Up to $96 \%$ of patients with CLN2 disease may have some form of sleep disorder (entire sleepless nights [78\%], difficulty falling asleep [69\%], drowsiness during the day [65\%], altered sleep duration [57\%], parasomnias [54\%], respiratory disorders during sleep [54\%], refusal to go to bed, anxiety, restless legs syndrome, regular leg movements) (19). As a result of insufficient sleep, (20) patients may develop mood disorders (depression, anxiety), fatigue, diurnal hypersomnia, reduced activity level, poor ictal control and other cognitive disorders that worsen the underlying cognitive problem (attention deficit, learning disabilities, memory consolidation deficit), with the ensuing variability in cognitive performance.

\section{Sleep: Recommended tools for use in patient follow-up}

\section{Clinical assessment and interview regarding sleep journal} and sleep disorder symptoms

- Frequency: At every visit

- History to be taken by: Neurologist/pediatric neurologist

- Recommendation grade: Mandatory.

- Observations: Documentation in the clinical record must include medical history (child development, sleep characteristics, school performance, school and social adaptation), use of medications and toxic substances, and physical examination. It is recommended to ask caregivers to keep a sleep journal.

\section{Sleep scales: PSQ (Pediatric Sleep Questionnaire)}

- Frequency: If sleep disorders are diagnosed, administer the questionnaire every 6 months. (21)

- Recommendation grade: Optional
- Scale to be administered by: Neurologist/pediatric neurologist. The caregiver may complete the questionnaire and bring it on the date of the visit.

- Validation: Validated in Colombia. Not validated in other countries.

- Observations: Includes all sleep disorders

\section{Actigraphy}

- Frequency: Whenever circadian cycle disorder is suspected, or if in doubt regarding sleep patterns, or when the information available is not very reliable.

- Test to be performed by: Sleep specialist

- Recommendation grade: Optional

\section{Polysomnography}

- Frequency: For confirmation purposes in cases of suspected sleep respiratory disorder (OSAHS), in restless legs syndrome and periodic limb movement disorder.

- Test performed by: Sleep specialist.

- Recommendation grade: Mandatory in case of suspected diagnosis.

\section{Pain: Clinical manifestations}

Patients with CLN2 disease may experience pain arising from multiple sources, with deleterious effects on their quality of life. Pain is primarily osteomuscular in advanced cases, and associated with some abnormal movements such as dystonia. Pain prevention and early identification must be an important management objective, as is the case in every neurodegenerative condition. $(3,22)$

\section{Pain: Recommended tools for use in patient follow-up}

Interview about the presence of pain and its characteristics

- Frequency: At every visit

- Interview to be conducted by: Neurologist/pediatric neurologist

- Recommendation grade: Mandatory

- Observations: It is not recommended to require caregivers to keep a journal

\section{Visual Analog Scale (VAS)}

- Frequency: At every visit, if there is pain of any type

- Scale to be administered by: It depends on patient age and condition. If the patient is cooperative, it may be completed by the patient or by the caregiver. Observation during the visit is key when the quality of the information is not adequate. No special training is required.

- Recommendation grade: Mandatory

- Observations: To assess the presence and severity of pain in cooperative patients, children over 6-8 years who are able to assess their pain. There is a recommendation for each age range in children $>3$ years. 
Behavioral scale or adapted from FLACC

- Frequency: At every visit, if there is pain of any type

- Scale to be administered by: If the patient is cooperative, it may be completed by the patient or by the caregiver. Observation during the visit is key when the quality of the information is not adequate. No special training is required.

- Recommendation grade: Mandatory

- Observations: For children aged 1 month to 4 years, uncooperative children, children with intellectual impairment, neurodevelopmental abnormalities or altered ability to communicate.

\section{Cognitive function follow-up}

\section{Clinical manifestations}

In patients with CLN2 disease there is a loss of psychomotor development milestones associated with epileptic seizures. Speech delay or regression (2-4 years of age) preceding epileptic seizures may be one of the first manifestations of the loss of cognitive functions. Following the onset of epileptic seizures, cognitive decline becomes evident and occurs at a faster rate. Altered behaviors such as agitation and anxiety are frequently present, creating distress for patients and family members alike. $(3,10)$

Studies have shown rapid cognitive decline (Hamburg and Weill Cornell scales). However, these tools only assess language and, more specifically, speech impairment. (23)

\section{Recommended tools for use in patient follow-up}

\section{Denver Developmental Screening Test (DENVER II Scale)} (24-26)

- Frequency: At baseline and every year from then on (children ages 0 to 6 )

- Scale to be administered by: Neuropsychologist or trained healthcare professional

- Recommendation grade: Mandatory

- Observations: Does not assess intelligence quotient, but it can be used to assess psychomotor development. In disease progression studies, assessment must be made every three months (Hamburg and Weill Cornell), due to the rapid decline in neurologic function.

Wechsler Intelligence Scale for Children (WISC IV) $(27,28)$

- Frequency: At baseline with yearly follow-up (children $>6$ years)

- Scale to be administered by: Neuropsychologist or trained healthcare professional (if approved for local use)

- Recommendation grade: Mandatory

- Validation: The scale has been validated in Spanish and Portuguese.

- The WISC-IV (Wechsler Intelligence Scale for Children IV) provides a broad assessment of cognitive functions. (29)

\section{Visual function follow-up}

\section{Clinical manifestations}

Visual involvement manifests slowly in children $>4$ years. Visual symptoms appear when cognitive decline and motor disorders are notorious. The onset of blindness typically occurs between 7 and 10 years of age. (3) Ophthalmological compromise occurs as a result of progressive retinal degeneration. In the final stages, there is diffuse atrophy secondary to progressive degeneration of the central nervous system involving projections of the visual pathways in the brain. The electroretinogram is often abnormal at the time of onset, even before retinal degeneration and changes in visual function are detectable. (30)

\section{Recommended tools for use in patient follow-up}

Anterior segment, eye fundus and visual acuity examination

- Frequency (experience-based recommendation): Annual follow-up

- Examination to be performed by: a) Fundus, ophthalmologist or neurologist; b) visual acuity, ophthalmologist or optometrist

- Recommendation grade: Mandatory

- Observations: The visual acuity test may be difficult to perform due to cognitive decline.

\section{Hamburg Visual Function Scale (31)}

- Frequency: At every visit

- Scale to be administered by: Pediatric neurologist/ neurologist or trained healthcare staff

- Recommendation grade (experience-based): Mandatory

- Validation: There is no validated translation in Spanish, but it is not needed

\section{Optic Coherence Tomography (OCT)}

- Frequency (experience-based recommendation): At baseline with yearly follow-up (depending on machine availability, patient cooperation and access to the test).

- Test to be performed by: Ophthalmologist

- Recommendation grade: Desirable

- Validation: Not needed. Use ophthalmic severity score (30)

- Observations: High-resolution OCT is adequate for detecting peripheral retinal changes and for determining the extent and progression of retinal abnormalities. The use of OCT will depend on the availability of the test at each healthcare institution.

\section{Electroretinogram (ERG)}

- Frequency: At baseline. Follow-up according to the course of the disease. (32)

- Test to be performed by: Trained visual electrophysiologist (standard international protocol) (33)

- Recommendation grade: Optional

- Observations: The use of ERG will depend on the availability of the test at each institution. 


\section{Follow-up of speech and language function}

\section{Clinical manifestations}

In classic late infantile CLN2 disease, language developmental delay, together with seizures, are the early manifestations of the disease. (34) Function decline (composite score), including language, occurs very rapidly (between 2 and 3 years of age). $(23,31)$ Progressive advanced neurological decline is associated with the deterioration of the swallowing function, which results in feeding problems that may require gastrostomy. Speech and language complications include delay in language and communication skills development, signs and symptoms of dysphagia, regression in language, dysarthria, swallowing abnormalities (rejection of certain foods, coughing during or immediately after eating or drinking), recurrent lower respiratory infections, weight gain or loss.

\section{Recommended tools for use in patient assessment:}

\section{Clinical assessment}

- Frequency (experience-based recommendation): At baseline and annual follow-up, as needed.

- Assessment to be performed by: Speech pathologist

- Recommendation grade: Mandatory

- Validation: Not required (local protocol)

- Observations: Monitoring for potential aspiration while on oral feeding.

\section{Hamburg (13) / Weill Cornell (31) scales for language assessment}

- Frequency (experience-based recommendation): At baseline and every 6 months during follow-up.

- Scale to be administered by: Neurologist/pediatric neurologist or trained healthcare staff

- Recommendation grade: Mandatory

- Validated: No validated translation is available in Spanish

\section{Instrumental assessment: Videofluoroscopic Swallow Study (VFSS) for swallowing assessment (35)}

- Frequency (experience-based recommendation): Only in children with suspected dysphagia or aspiration based on the clinical findings and the judgement of the speech pathologist.

- Test to be performed by: Speech pathologist and radiologist

- Recommendation grade (experience-based): Mandatory if dysphagia or airway penetration are suspected.

- Validation: Not required (local protocol)

\section{Cardiac function follow-up}

\section{Clinical manifestations $(36,37)$}

Cardiac complications and conduction abnormalities can be explained by the accumulation of lipopigments (ceroids and lipofuscin-like pigments) found not only in the neurons but in other tissues, including the heart. Cardiac findings are infrequent, although T-wave alterations and right ventricular hypertrophy have been described. There are no reports in the literature regarding cardiac manifestations before 14 years of age. There is one case description of CLN2 disease with biventricular dilation and severe heart failure but no definitive conclusion on whether the pathophysiological mechanisms of the disease may cause heart failure. (38)

\section{Recommended tools for use in patient assessment $(3,39)$}

\section{Pediatric cardiology consult}

- Frequency: At baseline and whenever cardiac manifestations occur.

- Patient to be seen by: Cardiologist

- Recommendation grade: Mandatory

\section{Electrocardiogram (ECG)}

- Frequency: At baseline with follow-up every two years (in the absence of cardiac findings). If abnormalities are found at the initial visit, follow-up every 6 months is recommended, based on the findings.

- Test to be performed by: Trained staff.

- Recommendation grade: Mandatory at the first visit and based on clinical findings from then on.

- Cardiovascular monitoring while on ERT: ECG monitoring must be done during the infusion in patients with a history of bradycardia, conduction disorder or structural heart disease, considering that some patients with CLN2 disease may develop conduction disorders or heart disease. In patients with normal cardiac function, regular 12-lead ECG assessments must be performed every 6 months.

\section{Echocardiography}

- Frequency: Follow-up every 2 years (in the absence of cardiac findings). If the initial echocardiogram is abnormal, follow-up every 6 months is recommended.

- Scan to be performed by: Cardiologist

- Recommendation grade: Mandatory at the first visit and based on clinical findings from then on.

\section{Holter}

- Frequency (experience-based): Every 6 months if altered rhythm is found.

- Test to be performed by: Cardiologist

- Recommendation grade: Desirable

\section{Quality-of-life (QoL) follow-up}

\section{Clinical manifestations}

CLN2 disease is a progressive, chronic, multi-system neurological condition in which cognitive, motor and sensorineural functions affected by the disease cannot be reverted with the treatments available at the present time. This translates into a significant impact on patient and family quality of life as a result of 
challenges with daily living which, to compound things, cannot be expressed during consultation time which is mostly devoted to the physical aspects of the disease. Maintaining communication, ensuring comfort and as much independence as possible for the patient, and supporting the family are important considerations.

\section{Recommended tools for use in patient assessment}

\section{Pediatric Quality of Life Scale (PedsQL 4.0) (40)}

- Frequency: Administer every 6 months unless important clinical or family changes occur.

- Recommendation grade: Mandatory

- Tool to be administered by: Trained professional (physician, psychologist, social assistant)

- Validation: Validated in all countries

- Observations: The scale may be administered to family members and caregivers or during the visit. Only children $>8$ years may be the main reporting party. It should not be administered too often in order to avoid adaptation. There is no agreement regarding the use of other scales in atypical adult patients.

\section{Motor function follow-up}

\section{Clinical manifestations}

In the juvenile form of CLN2 disease, cognitive, behavioral and motor symptoms are common and usually manifest after the onset of visual loss and seizures. In the late infantile form, these symptoms are preceded by language delay and rapidly progressing motor decline. The study of motor assessment in patients with CLN2 disease is limited. In its typical form, CLN2 disease has a devastating, rapidly progressing natural history and the clinical approach to this condition is limited to the use of the Hamburg and Weill Cornell scales as well as the CLN2 disease scale, based on the latter, and which was used in clinical studies for motor and language assessment. These are all gross scales for motor assessment, so functional losses or gains must be quite substantial to be reflected in higher or lower scores. Prior studies have used the 6-minute walk test, (40) with the limitation of requiring gait to be still present in order to conduct the assessment. Gross motor function assessment tools play a key role in the identification, diagnosis and assessment of motor challenges in early childhood. In their study, Griffiths A. et al. (41) carried out a systematic assessment of the psychometric properties and the clinical applicability of gross motor function assessment tools in children between 2 and 12 years of age, and showed that most of these tools have good-to-excellent validity. The Bayley-III has the best predictive validity at 2 years of age in terms of motor outcome later in life, but none of the tools showed good evaluative validity. Hence the need to develop a clear strategy for observation and follow-up which is reliable and validated in several languages, in order to ensure better follow-up. (41) In recent years, the Gross Motor Function Measure (GMFM) has become the most common functional outcome measure used by rehabilitation specialists for measuring gross motor function in children with cerebral palsy and other neurological conditions such as Down syndrome, traumatic head injury and osteogenesis imperfecta. The first version of the GMFM is the GMFM-88 and the most recent one is the GMFM-66.(42) Both versions are useful and valuable for assessing functional motor skills in children under 17 years of age. These tools have been translated and validated in several languages and used in different cultures. (42)

The GMFM is used by different rehabilitation specialists for clinical and research purposes. Considering its widespread use, its aim and the amount of research conducted using this tool, its use in motor assessment of children with CLN2 disease between 0 and 17 years of age is proposed.

\section{Recommended tools for use in patient assessment}

\section{Gross Motor Function Measure (GMFM)}

- Frequency: At baseline and every 6 or 12 months from then on, according to how the patient evolves.

- Scale to be administered by: Physical medicine and rehabilitation specialist, pediatric physiatrist, pediatric neurologist, physical therapist

- Recommendation grade: Mandatory

- Observations: Used to assess gross motor function in children with cerebral palsy and other motor disabilities.

- Validation: The Gross Motor Function scale is validated in Spanish and can be used in CLN2 disease

- Observations: The Gross Motor Function Classification System (GMFCS), plus the Manual Ability Classification System (MACS) questionnaire for upper limb assessment, may be used in centers that do not use the GMFM.

\section{Discussion and Conclusions}

CLN2 disease is the most common form of lipofuscinosis in South America, compared to the rest of the world. CLN2 disease with a slower or non-classical evolution, a later onset of symptoms and slower neurodegenerative progression versus the classical form, represents more than $50 \%$ of the cases of CLN2 disease described in the region, unlike the rest of the world. The mixture of races, with a wide genetic mix and the finding of different mutations and deletions observed in South America could explain this difference. (1-2)

Considering that there is a paucity of evidence regarding algorithms and protocols for follow-up of patients with CLN2 disease in Latin America, basic recommendations consistent with the characteristics of the Health Systems and access to, and availability of, resources and tools in the different countries have been developed for adequate patient follow-up based on the knowledge and experience of expert physicians in the region (Table 1).

Given the heterogeneity of the region, an attempt has been made to standardize logical and feasible criteria for both public and private healthcare facilities, taking into consideration diagnostic and follow-up resources. The fact that not all tools 
Table 1. Summary of recommendations for follow up in patients with CLN2 disease.

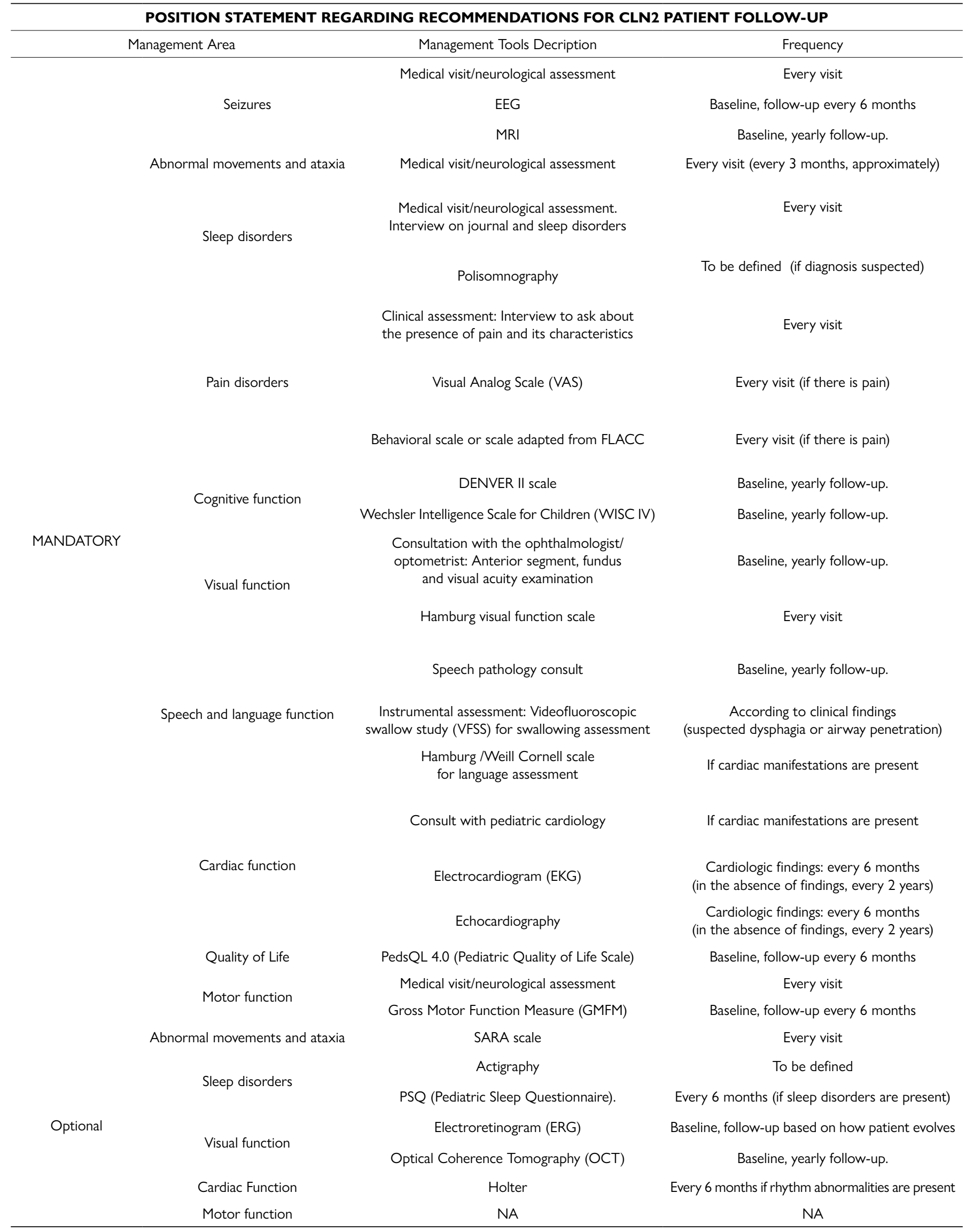


are validated in all countries is a challenge, but it is important to use the same tools if reliable results regarding the progression and follow-up of neuronal lipofuscinosis are to be obtained.

This position statement provides feasible follow-up guidance for the benefit of patients and treating physicians alike. It is derived from the views of expert professionals with experience in the management and follow-up of CLN2 disease.

The main challenge looking forward is to gain additional experience and access to the evidence needed to optimize management for the benefit of the patients and their families, focusing on improving the quality of life and wellbeing of those affected by the disease.

\section{Funding}

Financial support was received from Biomarin Pharmaceutical Inc. for the retainer of a medical writer. Biomarin Pharmaceutical, Inc. did not play any role in study design, data collection, analysis, data interpretation or writing of the report.

\section{Ethics Approval and Consent to Participate}

This article was written in compliance with copyright laws and conventions.

\section{Declaration of Conflicting Interests}

Guelbert N., Atanacio N., Denzler I., Embiruçu E. K., Mancilla N., Naranjo R., Tavera L., Troncoso M., and Vergara D., have received speaker and/or consulting honorarium from Biomarin.

\section{Authors' Contributions}

All authors participated on the conception, design, acquisition of data, manuscript writing, critical revision and final approval.

\section{Supplementary Material}

The following online material is available for this article: Annex 1 - Table of recommendations.

\section{References}

1. Kohan R, Pesaola F, Guelbert N, Pons P, Oller-Ramírez AM, Rautenberg G, et al. The neuronal ceroid lipofuscinoses program: A translational research experience in Argentina. Biochim Biophys Acta. 2015;1852(10 Pt B):2301-2311. doi: 10.1016/j.bbadis.2015.05.003

2. Kohan R, Carabelos MN, Xin W, Sims K, Guelbert N, Cismondi IA, et al. Neuronal ceroid lipofuscinosis type CLN2: A new rationale for the construction of phenotypic subgroups based on a survey of 25 cases in South America. Gene. 2013;516(1):114-121. doi: 10.1016/j.gene.2012.12.058
3. Williams RE, Adams HR, Blohm M, Cohen-Pfeffer JL, de los Reyes E, Denecke J, et al. Management Strategies for CLN2 Disease. Pediatr Neurol. 2017;69:102-112. doi: 10.1016/j.pediatrneurol.2017.01.034

4. Specchio N, Bellusci M, Pietrafusa N, Trivisano M, de Palma L, Vigevano F. Photosensitivity is an early marker of neuronal ceroid lipofuscinosis type 2 disease. Epilepsia. 2017;58(8):1380-1388. doi: 10.1111/epi.13820

5. Caraballo R, Sologuestua A, Ruggieri V.L, Monges S, Cersósimo R, Taratuto A, Medina C, Fejerman N. Clinical and electroencephalographic aspects of late infantile neuronal ceroid lipofuscinosis. Rev Neurol. 2005;40 :135140. doi: 10.33588/rn.4003.2003615

6. Dyke JP, Sondhi D, Voss HU, Shungu DC, Mao X, Yohay $\mathrm{K}$, et al. Assessment of disease severity in late infantile neuronal ceroid lipofuscinosis using multiparametric MR imaging. Am J Neuroradiol. 2013;34(4):884-889. doi: 10.3174/ajnr.A3297

7. Dyke JP, Sondhi D, Voss HU, Yohay K, Hollmann C, Mancenido $\mathrm{D}$, et al. Brain region-specific degeneration with disease progression in late infantile neuronal ceroid lipofuscinosis (CLN2 Disease). Am J Neuroradiol. 2016;37(6):1160-1169. doi: 10.3174/ajnr.A4669

8. Schulz A, Ajayi T, Specchio N, de Los Reyes E, Gissen P, Ballon D, et al. Study of intraventricular cerliponase alfa for CLN2 disease. N Engl J Med. 2018;378(20):1898-1907. doi: $10.1056 /$ nejmoa1712649

9. Beltrán L, reyes valenzuela G, Loos M, Vargas R, Spinzanti P, Lizama R, Caraballo R. Late-onset childhood neuronal ceroid lipofuscinosis: Early clinical and electroencephalographic markers. Epilepsy Res. 2018;144:49-52. doi: 10.1016/j. eplepsyres.2018.05.005

10. Mole SE, Williams RE, Goebel HH. Correlations between genotype, ultrastructural morphology and clinical phenotype in the neuronal ceroid lipofuscinoses. Neurogenetics. 2005;6:107-126. doi: 10.1007/s10048-0050218-3

11. Chang M, Cooper JD, Davidson BL, van Diggelen OP, Elleder M, Goebel HH, et al. CLN2. In: Mole S, Williams $\mathrm{R}$, and Goebel H eds. 2nd ed. The Neuronal Ceroid Lipofuscinoses (Batten Disease). Oxford University Press; 2011: 80-109. doi: 10.1016/s0022-510x(00)00289-6

12. Pérez-Poyato MS, Marfa MP, Abizanda IF, RodriguezRevenga L, Sánchez VC, González MJM, et al. Late infantile neuronal ceroid lipofuscinosis: Mutations in the CLN2 gene and clinical course in Spanish patients. J Child Neurol. 2013;28(4):470-478. doi: 10.1177/0883073812448459

13. Steinfeld R, Heim P, Von Gregory H, Meyer K, Ullrich $\mathrm{K}$, Goebel $\mathrm{HH}$, et al. Late infantile neuronal ceroid lipofuscinosis: Quantitative description of the clinical 
course in patients with CLN2 mutations. Am J Med Genet. 2002;112(4):347-354. doi: 10.1002/ajmg. 10660

14. Schulz A, Kohlschütter A, Mink J, Simonati A, Williams R. NCL diseases - clinical perspectives. Biochim Biophys Acta. 2013;1832(11):1801-1806. doi: 10.1016/j. bbadis.2013.04.008

15. Worgall S, Sondhi D, Hackett NR, Kosofsky B, Kekatpure M V., Neyzi N, et al. Treatment of late infantile neuronal ceroid lipofuscinosis by CNS administration of a serotype 2 adeno-associated virus expressing CLN2 cDNA. Hum Gene Ther. 2008;19(5):463-474. doi: 10.1089/hum.2008.022

16. Schmitz-Hübsch T, Du Montcel ST, Baliko L, Berciano J, Boesch S, Depondt C, et al. Scale for the assessment and rating of ataxia: Development of a new clinical scale. Neurology. 2006;66(11):1717-1720. doi: 10.1212/01. wnl.0000219042.60538.92

17. Krystkowiak P, Du Montcel ST, Vercueil L, Houeto JL, Lagrange C, Cornu P, et al. Reliability of the Burke-FahnMarsden scale in a multicenter trial for dystonia. Mov Disord. 2007;22(5):685-689. doi: 10.1002/mds.21392

18. Kurian MA, Dale RC. Movement Disorders Presenting in Childhood. Continuum (Minneap Minn). 2016;22(4):11591185:1159-1185. doi: 10.1212/con.0000000000000367

19. Lehwald LM, Pappa R, Steward S, De Los Reyes E. Neuronal Ceroid Lipofuscinosis and Associated Sleep Abnormalities. Pediatr Neurol. 2016;59:30-35. doi: 10.1016/j.pediatrneurol.2016.02.009

20. Owens J, Au R, Carskadon M, Millman R, Wolfson A, Braverman PK, et al. Insufficient sleep in adolescents and young adults: An update on causes and consequences. Pediatrics. 2014;134(3):e921-e932. doi: 10.1542/peds.20141696

21. San Miguel Montes LE, Allen DN, Puente AE, Neblina NC. Validity of the wisc-iv spanish for a clinically referred sample of hispanic children. Psychol Assess. 2010;22(2):465469. doi: 10.1037/a0018895.

22. Barney CC, Hoch J, Byiers B, Dimian A, Symons FJ. A casecontrolled investigation of pain experience and sensory function in neuronal ceroid lipofuscinosis. Clin J Pain. 2015;31(11):998-1003. doi: 10.1097/AJP.0000000000000192

23. Nickel M, Simonati A, Jacoby D, Lezius S, Kilian D, Van de Graaf B, et al. Disease characteristics and progression in patients with late-infantile neuronal ceroid lipofuscinosis type 2 (CLN2) disease: an observational cohort study. Lancet Child Adolesc Health. 2018;2(8):582-590. doi: 10.1016/S2352-4642(18)30179-2

24. Dos Santos RS, Araújo APQC, Porto MAS. Diagnóstico precoce de anormalidades no desenvolvimento em prematuros: Instrumentos de avaliação. J Pediatr (Rio J). 2008;84(4):289-299. doi: 10.2223/jped.1815
25. De-Andrés-Beltrán B, Rodríguez-Fernández ÁL, Güeita-Rodríguez J, Lambeck J. Evaluation of the psychometric properties of the Spanish version of the Denver Developmental Screening Test II. Eur J Pediatr. 2015;174(3):325-9. doi: 10.1007/s00431-014-2410-7

26. López-Pisón J, García-Jiménez MC, Monge-Galindo L, Lafuente-Hidalgo M, Pérez-Delgado R, García-Oguiza A, et al. Our experience with the aetiological diagnosis of global developmental delay and intellectual disability: 2006-2010. Neurol. 2014;29(7):402-407. doi: 10.1016/j. nrleng.2013.10.018

27. San Miguel Montes LE, Allen DN, Puente AE, Neblina NC. Validity of the wisc-iv spanish for a clinically referred sample of hispanic children. Psychol Assess. 2010;22(2):465469. doi: $10.1037 / \mathrm{a} 0018895$

28. Dias-Viana JL, Gomes GVA. Escala Wechsler de Inteligência para Crianças (WISC): análise da produção de artigos científicos brasileiros. Psicol Rev. 2019;28(1):9-36. doi: 10.23925/2594-3871.2019v28i1p9-36

29. Adams HR, Mink JW. Neurobehavioral features and natural history of juvenile neuronal ceroid lipofuscinosis (batten disease). J Child Neurol. 2013;28(9):1128-1136. doi: $10.1177 / 0883073813494813$

30. Orlin A, Sondhi D, Witmer MT, Wessel MM, Mezey JG, Kaminsky SM, et al. Spectrum of Ocular Manifestations in CLN2-Associated Batten (Jansky-Bielschowsky) Disease Correlate with Advancing Age and Deteriorating Neurological Function. PLoS One. 2013;8(8): e73128. doi: 10.1371/journal.pone.0073128

31. Worgall S, Kekatpure M V., Heier L, Ballon D, Dyke JP, Shungu D, et al. Neurological deterioration in late infantile neuronal ceroid lipofuscinosis. Neurology. 2007;69(6):521535. doi: 10.1212/01.wnl.0000267885.47092.40

32. Quagliato EMAB, Rocha DM, Sacai PY, Watanabe SS, Salomão SR, Berezovsky A. Retinal function in patients with the neuronal ceroid lipofuscinosis phenotype. Arq Bras Oftalmol. 2017;80(4):215-219. doi: 10.5935/00042749.20170053

33. Marmor MF, Fulton AB, Holder GE, Miyake Y, Brigell $M$, Bach M. ISCEV Standard for full-field clinical electroretinography (2008 update). Doc Ophthalmol. 2009;118(1):69-77. doi: 10.1007/s10633-008-9155-4

34. Fietz M, AlSayed M, Burke D, Cohen-Pfeffer J, Cooper JD, Dvořáková L, et al. Diagnosis of neuronal ceroid lipofuscinosis type 2 (CLN2 disease): Expert recommendations for early detection and laboratory diagnosis. Mol Genet Metab. 2016;119(1-2):160-167 doi: 10.1016/j.ymgme.2016.07.011

35. Batchelor G, Mcnaughten B, Bourke T, Dick J, Leonard C, Thompson A. How to use the videofluoroscopy swallow study in paediatric practice. Arch Dis Child 
Educ Pract Ed. 2019;104(6):313-320. doi: 10.1136/ archdischild-2017-313787

36. Østergaard JR, Rasmussen TB, Mølgaard H. Cardiac involvement in juvenile neuronal ceroid lipofuscinosis (Batten disease). Neurology. 2011;76(14):1245-51. doi: 10.1212/WNL.0b013e31821435bd

37. Hofman IL, van der Wal AC, Dingemans KP, Becker AE. Cardiac pathology in neuronal ceroid lipofuscinosesa clinicopathologic correlation in three patients. Eur J Paediatr Neurol. 2001;5(Suppl A):213-217. doi: 10.1053/ ejpn.2000.0465

38. Khanji MY, Rusinova R, Shaukat M, Sekhri N. Neuronal ceroid lipofuscinosis with severe biventricular impairment: A rare genetic disorder with associated myopathy? Eur Heart Journal. 2018. 39(1):74. doi: 10.1093/eurheartj/ ehx691

39. Kusumoto FM, Schoenfeld MH, Barrett C, Edgerton JR, Ellenbogen KA, et al. 2018 ACC/AHA/HRS Guideline on the Evaluation and Management of Patients With
Bradycardia and Cardiac Conduction Delay: A Report of the American College of Cardiology/American Heart Association Task Force on Clinical Practice Guidelines and the Heart Rhythm Society. Circulation. 2019;140:e382-e482. doi: 10.1542/peds.2006-2099

40. Varni JW, Seid M, Kurtin PS. PedsQL ${ }^{\mathrm{TM}}$ 4.0: Reliability and Validity of the Pediatric Quality of Life Inventory ${ }^{\mathrm{TM}}$ Version 4.0 Generic Core Scales in Healthy and Patient Populations. Med Care. 2001;39(8):800-812. doi: 10.1097/00005650200108000-00006

41. Griffiths A, Toovey R, Morgan PE, Spittle AJ. Psychometric properties of gross motor assessment tools for children: a systematic review. BMJ Open. 2018;8(10):e021734. doi: 10.1136/bmjopen-2018-021734

42. Alotaibi M, Long T, Kennedy E, Bavishi S. The efficacy of GMFM-88 and GMFM- 66 to detect changes in gross motor function in children with cerebral palsy (CP): a literature review. Disabil Rehabil. 2014;36(8):617-627. doi: 10.3109/09638288.2013.805820 